\title{
Serum tenascin- $C$ discriminates patients with active SLE from inactive patients and healthy controls and predicts the need to escalate immunosuppressive therapy: a cohort study
}

\author{
Jakub Závada1*, Michal Uher², Radka Svobodová', Marta Olejárová', Markéta Hušáková1, Hana Ciferská1, \\ Hana Hulejová ${ }^{\prime}$, Michal Tomčík ${ }^{1}$, Ladislav Šenolt ${ }^{1}$ and Jiří Vencovský ${ }^{1}$
}

\begin{abstract}
Introduction: The aim of this study was to examine whether circulating levels of the proinflammatory glycoprotein tenascin-C (TNC) are useful as an activity-specific or predictive biomarker in systemic lupus erythematosus (SLE).

Methods: Serum TNC levels were determined by enzyme-linked immunosorbent assay at inception visit in a prospective cohort of 59 SLE patients, and in 65 healthy controls (HC). SLE patients were followed for a mean of 11 months, disease activity was assessed using the Systemic Lupus Erythematosus Disease Activity Index 2000 (SLEDAI-2 K) and British Isles Lupus Assessment Group disease activity index (BILAG-2004), clinical and laboratory data were recorded every 3-6 months, and changes in glucocorticoids (GC) and immunosuppressants (IS) were recorded serially. We examined cross-sectionally the relationships between serum concentrations of TNC and SLE status, SLEDAI-2 K scores, strata of disease activity, and levels of conventional biomarkers [anti-double-stranded DNA (dsDNA), anti-nucleosome antibodies, C3 and C4]. We also explored the utility of TNC levels for predicting disease flares, defined as (i) new/increased GC, (ii) new/increased GC or IS, and (iii) increase in SLEDAI by $\geq 3$ or (iv) BILAG A or B flare.
\end{abstract}

Results: There was no significant difference in the mean levels of TNC between the SLE patients and HC. However, in SLE patients with active disease (SLEDAI $\geq 6)$, the TNC levels were significantly higher than in the HC $(p=0.004)$ or in patients with no/low disease activity $(p=0.004)$. In SLE patients, TNC levels were significantly associated with positivity of anti-dsDNA ( $p=0.03$ ) and anti-nucleosome antibodies $(p=0.008)$. Flares defined by a need to escalate immunosuppressive therapy were captured more frequently and earlier than flares defined by standard activity indices. Higher baseline levels of serum TNC presented a significantly greater risk of flare (i) [hazard ratio (HR) 1.39, $95 \%$ confidence interval (Cl) 1.11-1.73] or (ii) (HR 1.25, $95 \%$ Cl 1.02-1.52) but not of flares (iii) or (iv). The baseline serum TNC level was the single most important independent predictor of flare (i) compared with conventional biomarkers.

Conclusions: TNC is not disease-specific, but it seems to indicate the activity of SLE and may predict the need to escalate immunosuppressive therapy. TNC levels may thus serve as a useful activity-specific and predictive biomarker in SLE.

Keywords: Tenascin-C, Biomarker, SLE, Disease activity, Flare

\footnotetext{
* Correspondence: zavada@revma.cz

'Institute of Rheumatology, Prague, and Department of Rheumatology, First Faculty of Medicine, Charles University in Prague, Na Slupi 4, Praha 2, 12850 Prague, Czech Republic

Full list of author information is available at the end of the article
} 


\section{Introduction}

Systemic lupus erythematosus (SLE) is a chronic autoimmune disease characterized by a wide range of manifestations involving nearly all organs, including the skin, kidney, lung, brain, heart and joints. Its precise aetiopathogenesis remains unclear. Diverse serological and clinical manifestations as well as unpredictable flares and remissions are observed among patients with SLE, and they present a challenge for the evaluation of disease activity and administration of appropriate treatment. Although clinical assessment is the cornerstone of management of patients with SLE, these evaluations are limited and require additional instruments to confirm the diagnosis and determine disease activity.

Traditional serological biomarkers such as anti-dsDNA antibodies and complement levels have been proven to be neither reliable indicators of disease activity $[1,2]$ nor predictors of impending disease flares [3]. The lack of useful biomarkers for SLE hampers assessment of disease activity and impedes the evaluation of treatment response. For this reason, there is growing interest in the exploration of new biomarkers for use as surrogate markers of disease activity and/or to predict flares of the disease.

Tenascin-C (TNC) is a large extracellular matrix glycoprotein that belongs to the damage-associated molecular patterns family [4]. Little TNC is found in most healthy adult tissues, because it is specifically induced and tightly controlled during acute inflammation and persistently expressed during chronic inflammation [5-11]. The induction of TNC is highly associated with a wide range of diseases related to inflammation, including pneumonitis [12], hepatitis [13], inflammatory bowel disease [14], myocarditis [15], atherosclerosis [16], obesity [17], rheumatoid arthritis [18] and the enthesitis-related arthritis category of juvenile idiopathic arthritis [19]. An early inflammatory response is generally associated with enhanced TNC levels, both in the plasma and in tissue [6]. Thus, plasma levels of TNC have been shown to be useful indicators for chronic hepatitis C [13], inflammatory bowel disease [14] and myocarditis [15].

To date, no researchers have reported whether circulating TNC levels could reflect disease activity and/or early tissue damage in SLE. Using clinical and laboratory data from our prospective cohort of patients with SLE, we investigated the association of serum TNC levels with Systemic Lupus Erythematosus Disease Activity Index 2000 (SLEDAI-2 K) scores and conventional laboratory markers of disease activity, such as antidouble-stranded DNA antibodies (anti-dsDNA), C3, C4 and anti-nucleosome antibodies. Moreover, we tested the clinical utility of serum TNC levels for the identification of patients with active disease and the prediction of disease flares.

\section{Material and methods}

\section{Subjects and data collection}

Fifty-nine patients fulfilling the revised 1997 American College of Rheumatology classification criteria for SLE [20] were recruited from the Institute of Rheumatology, Prague, and prospectively followed according to a predefined protocol. Clinical and laboratory assessments were performed at baseline, after 3 months, after 6 months and every 6 months thereafter. At each per-protocol visit, disease activity was assessed using the SLEDAI-2 K [21] and the British Isles Lupus Assessment Group disease activity index (BILAG-2004) [22], and the current dosage of glucocorticoids (GC) and each immunosuppressant (IS) medication prescribed for SLE were recorded, in addition to previous changes in medication updated from the source documentation. Patients were followed longitudinally for a mean of 10.8 months [standard deviation (SD) 6.5]. Healthy controls $(\mathrm{HC} ; \mathrm{n}=65)$ were also recruited. All study participants were $\geq 18$ years of age, and each participant provided written informed consent. The study design and written consent were approved by our institution's ethics committee.

\section{Definitions}

Because we were interested mainly in global SLE activity, our primary outcome definitions were based on the SLEDAI-2 K. The scores for the clinical items of the SLEDAI-2 K (c-SLEDAI-2 K) were calculated by subtracting the contribution of hypocomplementaemia and anti-dsDNA positivity from the total SLEDAI- $2 \mathrm{~K}$ score. Active disease was defined as a SLEDAI- $2 \mathrm{~K}$ score $\geq 6$ [23]. A disease flare was defined as (i) new/increased GC, (ii) new/increased GC or IS, (iii) an increase in the SLEDAI-2 $K \geq 3$ and (iv) BILAG-2004 A or B flare. For analytical purposes, SLEDAI- $2 \mathrm{~K}$ items describing the involvement of one organ or tissue were consolidated into a single SLEDAI-2 K domain (neuropsychiatric features $=$ seizure, psychosis, organic brain syndrome, cranial nerve disorder, lupus headache, cerebrovascular accident; renal features = haematuria, proteinuria, pyuria, urinary casts; serositis = pleurisy, pericarditis; haematological features $=$ thrombocytopenia, leukopenia).

\section{Laboratory analysis}

Fasting blood samples were collected from all patients during the baseline visit. The samples were immediately centrifuged and stored at $-80{ }^{\circ} \mathrm{C}$. Levels of TNC in the serum samples were determined using the human TNC Large (FN III-B) Assay enzyme-linked immunosorbent assay (ELISA) kit from IBL (Fujioka, Japan). The samples were diluted 400-fold. The absorbance was measured using an ELISA reader (SUNRISE; Tecan, Grödig, Austria) using $450 \mathrm{~nm}$ as the primary wavelength. The intra- and inter-assay coefficients of variation for this ELISA were $6.4 \%$ and $3.5 \%$, 
respectively, at concentrations of $5.43 \mathrm{ng} / \mathrm{ml}$ and $6.55 \mathrm{ng} / \mathrm{ml}$. The sensitivity for this kit was $44 \mathrm{pg} / \mathrm{ml}$.

The routine laboratory and immunological measurements needed for the calculation of the SLEDAI-2 K were performed at every visit, and other routine immunological tests [anti-nuclear antibodies (ANA), ANA line immunoassay (LIA) and anti-nucleosome antibodies) were measured at the baseline visit. ANA antibodies were detected by indirect immunofluorescence (Immuno Concepts, Sacramento, CA, USA) and further characterized by the LIA method (IMTEC, Wiesbaden, Germany). AntidsDNA antibodies were detected by immunofluorescence (Immuno Concepts); normal was defined as a negative titre. Anti-nucleosome antibodies were measured by ELISA (EUROIMMUN, Lübeck, Germany); normal was defined as $0-24 \mathrm{U} / \mathrm{ml}$. Complement levels were measured using the AU system with reagents (Beckman Coulter, Brea, CA, USA). The reference range for $\mathrm{C} 3$ in the serum was $0.9-1.8 \mathrm{~g} / \mathrm{L}$, and for $\mathrm{C} 4$ it was $0.1-0.4 \mathrm{~g} / \mathrm{L}$.

\section{Statistical analysis}

Continuous variables were expressed as the mean \pm SD . Categorical data were summarized as absolute frequencies and percentages. We used univariate and multivariable (with adjustments for age and sex) linear regression analyses to assess the association between the baseline serum TNC levels and clinical and laboratory manifestations of SLE. We derived survival curves using the Kaplan-Meier method, and Cox proportional hazards models were used to analyse the association between baseline serum TNC levels and prospectively measured indicators of disease flares. The results were presented as hazard ratio (HR) and $95 \%$ confidence interval (CI). Serum TNC levels were analysed as a continuous variable, with HRs calculated per $100 \mathrm{ng} / \mathrm{ml}$ increment of serum TNC. The relationship between serum TNC levels and the risk of endpoint was examined in unadjusted and multivariable adjusted Cox models. The Akaike information criterion (AIC) was used to compare the ability of each immunological marker to predict disease flares, and the differences between predictive models were tested using the likelihood ratio test. Receiver operating characteristic (ROC) curve analysis was performed using data from the inception visit to establish the optimal discriminatory threshold to identify patients with active disease (defined as SLEDAI-2 $\mathrm{K} \geq 6$ ) and to predict (i) flares based on TNC levels. A two-tailed $p$ value $<0.05$ was considered statistically significant. Statistical analyses were performed using IBM SPSS version 22 software (IBM SPSS, Armonk, NY, USA).

\section{Results}

Demographic and clinical characteristics of patients with SLE The SLE cohort consisted of $93 \%$ women with a mean $( \pm \mathrm{SD})$ age of $44 \pm 16$ years. At baseline, $95 \%$ were
ANA-positive, $39 \%$ were anti-dsDNA-positive, $46 \%$ were anti-nucleosome antibody-positive and $49 \%$ had low serum complement (C3, C4 or both). The mean $( \pm \mathrm{SD})$ SLEDAI-2 $\mathrm{K}$ was $3.7 \pm 3.5$, the disease duration was $7 \pm 7$ years, $33 \%$ had active disease as defined by a SLEDAI- $\mathrm{K} \geq 6,59 \%$ were using oral GC, $42 \%$ were using anti-malarials and $20 \%$ were using IS. The $\mathrm{HC}$ were somewhat older, and males were more often represented. The baseline characteristics of the SLE cohort and the demographics of the $\mathrm{HC}$ are summarized in Table 1.

Table 1 Baseline characteristics

\begin{tabular}{|c|c|c|}
\hline & SLE $(n=59)$ & $\begin{array}{l}\text { Healthy controls } \\
(n=65)\end{array}$ \\
\hline Female sex & $55(93 \%)$ & $45(69 \%)$ \\
\hline Age, yr & $44 \pm 16$ & $48(14)$ \\
\hline Caucasian & $59(100 \%)$ & $65(100 \%)$ \\
\hline Disease duration, yr & $7 \pm 7$ & \\
\hline SLICC/ACR Damage Index & $0.8 \pm 1.4$ & \\
\hline SLEDAI-2 K & $3.7 \pm 3.5$ & \\
\hline cSLEDAI-2 K (only clinical SLEDAI-2 K items) & $2.2 \pm 3.0$ & \\
\hline SLEDAI- $2 \mathrm{~K} \geq 4$ & $30(53 \%)$ & \\
\hline SLEDAI-2 K $\geq 6$ & $19(33 \%)$ & \\
\hline Any SLEDAI-2 K clinical features & $26(45 \%)$ & \\
\hline Neuropsychiatric features ${ }^{\mathrm{a}}$ & $2(3 \%)$ & \\
\hline Vasculitis $^{\mathrm{a}}$ & $0(0 \%)$ & \\
\hline Arthritis $^{a}$ & $10(17 \%)$ & \\
\hline Myositis $^{a}$ & $0(0 \%)$ & \\
\hline Renal features ${ }^{a}$ & $8(14 \%)$ & \\
\hline Rash $^{a}$ & $9(15 \%)$ & \\
\hline Alopecia $^{a}$ & $5(9 \%)$ & \\
\hline Mucosal ulcers ${ }^{a}$ & $0(0 \%)$ & \\
\hline Serositis $^{a}$ & $1(2 \%)$ & \\
\hline Haematological features $^{\mathrm{a}}$ & $3(5 \%)$ & \\
\hline Fever $^{a}$ & $0(0 \%)$ & \\
\hline Increased DNA binding ${ }^{a}$ & $22(38 \%)$ & \\
\hline Low complement ${ }^{\mathrm{a}}$ & $28(48 \%)$ & \\
\hline Anti-nucleosome antibody-positive & $25(46 \%)$ & \\
\hline Oral glucocorticoids & $35(59 \%)$ & \\
\hline Immunosuppressants & $15(25 \%)$ & \\
\hline
\end{tabular}

ANA anti-nuclear antibodies, SLEDAl-2 K Systemic Lupus Erythematosus Disease Activity Index 2000, anti-dsDNA anti-double-stranded DNA, SLICC/ACR Systemic Lupus International Collaborating Clinics/American College of Rheumatology, SLE systemic lupus erythematosus

Data are presented as number and percentage or mean and standard deviation

${ }^{\mathrm{a}}$ According to SLEDAl-2 K definitions; renal, haematological, serositis and neuropsychiatric SLEDAI-2 K features were merged into one item (see Definitions section in text) 


\section{Serum TNC levels in patients with SLE and healthy controls}

There was no significant difference in the mean levels of TNC between the patients with SLE and HC (533 \pm $192 \mathrm{ng} / \mathrm{ml}$ vs. $487 \pm 164 \mathrm{ng} / \mathrm{ml}, p=0.151)$. However, in patients with SLE with active disease (SLEDAI-2 $K \geq 6$ ) the TNC levels were significantly higher than in the HC $(634 \pm 254 \mathrm{ng} / \mathrm{ml}$ vs. $487 \pm 164 \mathrm{ng} / \mathrm{ml}, p=0.004)$ or patients with no or low disease activity $(634 \pm 254 \mathrm{ng} / \mathrm{ml}$ vs. $481 \pm$ $135 \mathrm{ng} / \mathrm{ml}, p=0.004$ ) (Fig. 1). We found no association between age, sex and TNC levels (see Additional file 1).

\section{Association between circulating levels of TNC and measures of disease activity}

A cross-sectional correlation and univariate linear regression analysis between serum TNC levels and SLEDAI-2 K $(\beta=14,95 \% \mathrm{CI}-1$ to $29, r=0.25, p=0.061)$ and $c-$ SLEDAI-2 K ( $\beta=16,95 \% \mathrm{CI}-0.7$ to $33, r=0.25, p=0.060)$ at baseline visit showed a trend towards a positive correlation, although the results were not statistically significant. Patients with SLE with active involvement of at least one SLEDAI-2 K domain (see Definitions section) have significantly higher TNC levels than patients with no clinical involvement according to SLEDAI-2 K $(\beta=108,95 \% \mathrm{CI}$ $8-207, p=0.035)$, and patients with any active item in the renal SLEDAI-2 $\mathrm{K}$ domain had significantly higher TNC levels than patients without active renal SLEDAI-2 K features (see Table 2 and Additional file 2). The crosssectional associations between TNC and BILAG-2004 organ domains are shown in Additional file 2. (Note that the renal BILAG-2004 domain could not be properly assessed at inception visit, while its evaluation is heavily

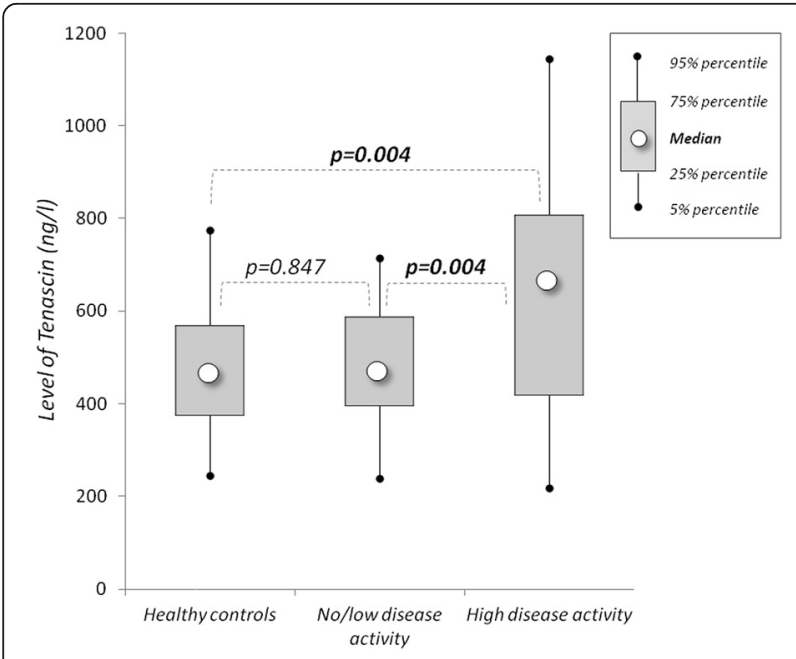

Fig. 1 Mean levels of tenascin- $C$ in healthy controls and patients with systemic lupus erythematosus with low [Systemic Lupus Erythematosus Disease Activity Index 2000 (SLEDAI-2 K) <6] and high (SLEDAl-2 K $\geq 6$ ) disease activity dependent on previous measurements which were not captured in our database.)

\section{Serum TNC levels discriminate between active and inactive disease}

ROC curve analysis was performed to establish the optimal discriminatory threshold to identify patients with active disease (defined as SLEDAI-2 $\mathrm{K} \geq 6$ ) based on TNC levels (Fig. 2). At the optimal cutoff point of $659 \mathrm{ng} / \mathrm{ml}$, the area under the curve for TNC serum levels that discriminated between active and inactive disease was 0.69 (95\% CI 0.53-0.86, $p=0.02$ ) with a sensitivity of $53 \%$ and specificity of $92 \%$.

\section{Association between circulating levels of TNC and conventional laboratory parameters}

A cross-sectional univariate linear regression and/or correlation analysis between serum TNC levels and other laboratory measurements in patients with SLE at the initial visit was performed (Table 2 and Additional file 3a, b). The TNC levels in patients with SLE were positively correlated with positivity for anti-dsDNA antibodies $(\beta=115$, $95 \%$ CI 12-218, $p=0.029)$ and anti-nucleosome antibodies $(\beta=138,95 \%$ CI 38-238, $p=0.008)$. Associations between TNC and these parameters were statistically significant even after adjustment for age and sex. In contrast, we did not find a significant association between serum TNC levels and C3/C4 levels.

\section{Serum TNC levels are predictive of disease flares}

Flares defined by a need to escalate immunosuppressive therapy [(i), (ii)] were captured more frequently and earlier than flares defined by standard activity indices [(iii), (iv)] (Fig. 3). Higher levels of serum TNC presented a significantly greater risk of flare (i) (defined as the need to start or escalate GC) (HR 1.39, $95 \%$ CI 1.11-1.73) or (ii) (defined as the need to start or escalate any IS) (HR 1.25, $95 \%$ CI 1.02-1.52), but not of flare (iii) (defined as an increase in SLEDAI-2 K $\geq 3$ ) (HR 1.19, 95 \% CI 0.871.63) or (iv) defined as a BILAG-2004 flare A or B (Table 3).

We also conducted a separate analysis in which serum TNC levels were treated as a categorical variable. We used the value of $659 \mathrm{ng} / \mathrm{ml}$ from the previous ROC curve analysis as a cutoff to identify patients with active disease. This value was almost identical to the value of $654 \mathrm{ng} / \mathrm{ml}$ generated by a separate ROC curve analysis to find an optimal discriminatory threshold to identify patients predicted to experience flare (i). In accordance with the result above, the risk of flare (i) or (ii) was significantly higher in the group of patients with higher TNC levels (Table 3, Fig. 4). 
Table 2 Cross-sectional associations between serum TNC levels and clinical and laboratory parameters of patients with SLE at the inception visit (univariate and age- and sex-adjusted regression analyses)

\begin{tabular}{|c|c|c|c|c|}
\hline \multirow[b]{2}{*}{ Variable } & \multicolumn{2}{|l|}{ Univariate analyses } & \multicolumn{2}{|c|}{ Age- and sex-adjusted analyses } \\
\hline & $\beta$ value $^{\mathrm{b}}(95 \% \mathrm{Cl})$ & $p$ Value & $\beta$ value $^{\mathrm{b}}(95 \% \mathrm{Cl})$ & $p$ Value \\
\hline Patients with SLE vs. HC & $46(-17$ to 110$)$ & 0.151 & $44(-24$ to 112$)$ & 0.205 \\
\hline Patients with SLE with SLEDAI- $2 \mathrm{~K} \geq 6$ vs. HC & $147(50-245)$ & 0.004 & $139(34-245)$ & 0.010 \\
\hline Patients with SLE with SLEDAI- $2 \mathrm{~K} \geq 6$ vs. SLEDAI- $2 \mathrm{~K}<6$ & $153(50-256)$ & 0.004 & $161(54-267)$ & 0.004 \\
\hline \multicolumn{5}{|l|}{ Patients with SLE (categorical variables) } \\
\hline Any SLEDAI-2 K clinical features (yes vs. no) & $107.8(8.1-207.4)$ & 0.035 & $108.4(3.6-213.3)$ & 0.043 \\
\hline Neuropsychiatric clinical features ${ }^{a}$ (yes vs. no) & $-211.9(-488.9$ to 65.2$)$ & 0.131 & $-202.5(-485.8$ to 80.8$)$ & 0.158 \\
\hline Vasculitis $^{\mathrm{a}}$ (yes vs. no) & - & - & - & - \\
\hline Arthritis $^{\mathrm{a}}$ (yes vs. no) & $48.3(-87.7$ to 184.2$)$ & 0.480 & $46.0(-94.4$ to 186.4$)$ & 0.514 \\
\hline Myositis (yes vs. no) & - & - & - & - \\
\hline Renal features ${ }^{\mathrm{a}}$ (yes vs. no) & $265.2(133.5-396.9)$ & $<0.001$ & $269.7(135.9-403.5)$ & $<0.001$ \\
\hline Rash $^{\mathrm{a}}$ (yes vs. no) & $-2.1(-144.6$ to 140.4$)$ & 0.977 & $-9.1(-154.9$ to 136.6$)$ & 0.901 \\
\hline Alopecia $^{a}$ (yes vs. no) & $-84.6(-267.0$ to 97.8$)$ & 0.357 & $-85.0(-270.7$ to 100.7$)$ & 0.363 \\
\hline Mucosal ulcers ${ }^{\mathrm{a}}$ (yes vs. no) & - & - & - & - \\
\hline Serositis ${ }^{\mathrm{a}}$ (yes vs. no) & $231.1(-160.4$ to 622.6$)$ & 0.242 & $216.6(-187.5$ to 620.7$)$ & 0.287 \\
\hline Haematological features ${ }^{a}$ (yes vs. no) & $20.4(-212.5$ to 253.3$)$ & 0.861 & 9.1 ( -231.3 to 249.5$)$ & 0.940 \\
\hline Fever $^{\mathrm{a}}$ (yes vs. no) & - & - & - & - \\
\hline Anti-dsDNA antibodies IF (positive vs. negative) & $115(12-218)$ & 0.029 & $112(3-221)$ & 0.044 \\
\hline Complement C3/C4 (low vs. normal) & $-4(-107$ to 99$)$ & 0.938 & $-14(-123$ to 94$)$ & 0.793 \\
\hline Anti-nucleosome antibodies (positive vs. negative) & $138(38-238)$ & 0.008 & $131(30-234)$ & 0.013 \\
\hline \multicolumn{5}{|l|}{ Patients with SLE (continuous variables) } \\
\hline SLEDAI-2 K & $14(-1$ to 29$)$ & 0.061 & $14(-1.5$ to 30$)$ & 0.074 \\
\hline cSLEDAI-2 K (only clinical SLEDAl-2 K items) & $16(-1$ to 33$)$ & 0.060 & $16(-1.0$ to 34$)$ & 0.065 \\
\hline $\mathrm{C} 3, \mathrm{~g} / \mathrm{L}$ & $-9(-209$ to 192$)$ & 0.931 & $5(-205$ to 216$)$ & 0.958 \\
\hline $\mathrm{C} 4, \mathrm{~g} / \mathrm{L}$ & $-232(-695$ to 230$)$ & 0.319 & $-210(-694$ to 273$)$ & 0.386 \\
\hline Anti-nucleosome antibodies, $U$ & $-0.2(-0.7$ to 0.3$)$ & 0.403 & $-0.2(-0.7$ to 0.3$)$ & 0.460 \\
\hline Anti-dsDNA antibodies, titre & $27(-22$ to 75$)$ & 0.266 & $30(-21$ to 81$)$ & 0.230 \\
\hline Urinary protein/creatinine ratio, mg/mmol & $270(101-439)$ & 0.002 & $271(98-444)$ & 0.003 \\
\hline
\end{tabular}

Cl confidence interval, $\mathrm{HC}$ healthy controls, IF immunofluorescence, anti-dsDNA anti-double-stranded DNA, SLEDAl-2 K Systemic Lupus Erythematosus Disease Activity Index 2000

${ }^{a}$ According to SLEDAI-2 K definitions; renal, haematological, serositis and neuropsychiatric SLEDAl-2 K features were merged into one item (see Definitions section of text) ${ }^{b}$ The regression coefficient $\beta$ corresponds to the difference in TNC levels between groups (when assessing categorical variables) or to the change in TNC associated with a 1 unit increase in the assessed variable (when assessing continuous variables)

Boldface type indicates statistically significant values

\section{Serum TNC levels outperformed traditional biomarkers in the prediction of flare (i)}

Next, we compared the performance of univariate models based either on traditional biomarkers (i.e., anti-dsDNA or anti-nucleosome antibodies and $\mathrm{C} 3$ or $\mathrm{C} 4$ ) or serum TNC levels to predict flare (i) using the AIC. As shown in Table 4, serum TNC levels outperformed traditional biomarkers when treated as both a continuous variable and a categorical variable.

\section{Discussion}

In this study, we found that high TNC levels reflected disease activity and predicted the escalation of GC or other immunosuppressive therapies. To the best of our knowledge, this is the first study to examine in detail the role of TNC in patients with SLE.

An important prerequisite for testing a biomarker in a new clinical setting is its biological plausibility. SLE is a complex autoimmune disease characterized by enhanced autoantibody formation, excessive proinflammatory cytokine production and damage to multiple organ systems. TNC is a proinflammatory extracellular matrix glycoprotein that has been shown to be involved in the regulation of both innate and adaptive immune systems and to control the expression of various cytokines and the recruitment of immune cells to sites of inflammation or injury 


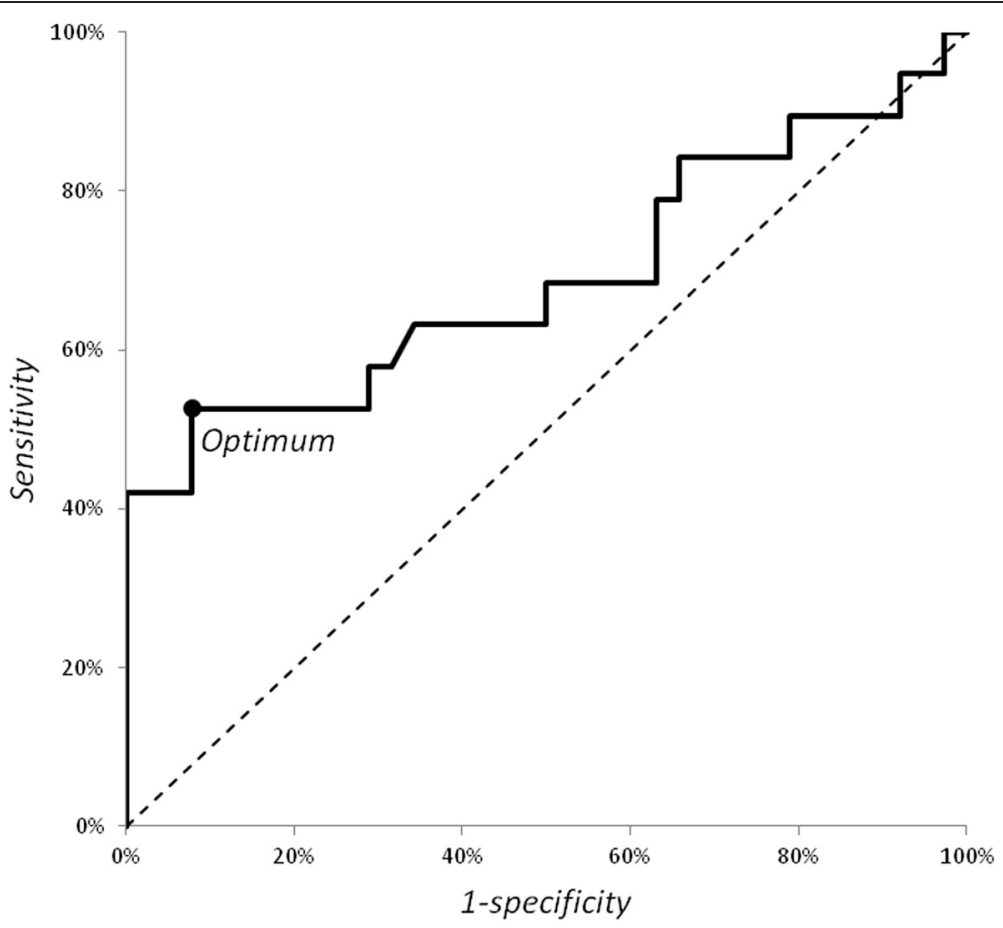

Fig. 2 Receiver operating characteristic curve analysis of tenascin (TNC) serum levels as a predictor of active systemic lupus erythematosus (defined as Systemic Lupus Erythematosus Disease Activity Index $2000 \geq 6$ ). At the optimal cutoff point of 659 ng/ml, the area under the curve for TNC serum levels that discriminated between active and inactive disease was 0.69 (95 \% Cl 0.53-0.86, p=0.02) with a sensitivity of 53 \% and specificity of $92 \%$

[7-11]. Little or no TNC is found in most healthy adult tissues, but it is upregulated under pathological conditions accompanying tissue injury and inflammation in many different organs that may be involved in the SLE disease process, including the joints [8, 18], skin [24], kidney [25], lungs [26], heart [27] and central nervous system [28]. Therefore, TNC seems to be an eligible candidate surrogate marker of ongoing tissue damage and may reflect disease activity or an impending flare. Moreover, TNC expression was previously shown to have some applications in disease diagnosis and outcome prognostication in immune-mediated and other inflammatory diseases [7-19, 24-28].

The initial cross-sectional correlation analyses appeared to support the utility of TNC as a putative SLE biomarker. At the inception visit in our SLE cohort, TNC levels were significantly associated with positivity of anti-dsDNA and anti-nucleosome antibodies. Additionally, there was a trend towards a positive correlation with the SLEDAI-2 K and c-SLEDAI-2 K scores. Interestingly, patients with SLE with active renal involvement (mainly with proteinuria) had significantly higher TNC levels than other patients with SLE. This finding is in line with other observations of increased local expression or increased TNC circulating levels in patients with different types of renal disease [29-31].
During the process of validation of a biomarker for the assessment of disease activity, the first external criterion to be correlated with the biomarker may be dichotomous (i.e., active vs. inactive SLE) [32]. To delineate active disease from inactive disease, we chose the robust definition of SLEDAI- $2 \mathrm{~K} \geq 6$ that has been used in most clinical SLE trials [23]. We found that mean TNC levels in patients with SLE with active disease were significantly higher than those of patients with SLE with no or low disease activity or those of HC. High serum TNC levels showed $53 \%$ sensitivity and $92 \%$ specificity in a ROC curve analysis. Hence, TNC levels could be a useful marker for distinguishing active from inactive SLE.

We also investigated the ability of serum TNC levels to predict disease flares. Higher baseline levels of serum TNC showed a significantly greater risk of a disease flare, defined as the need to start or escalate GC or other immunosuppressive therapy but not a flare defined as a change in the SLEDAI-2 K $\geq 3$ or a BILAG-2004 A or B flare. There is no clear consensus on the best definition of a flare in SLE or on the minimal clinically important change that would be both sensitive and specific [33]. We used four definitions to define disease flares: two based on a change in immunosuppressive therapy [(i) and (ii)] and two based on a change in SLEDAI-2 K or BILAG-2004 [(iii) and (iv)]. The Kaplan-Meier curves in 


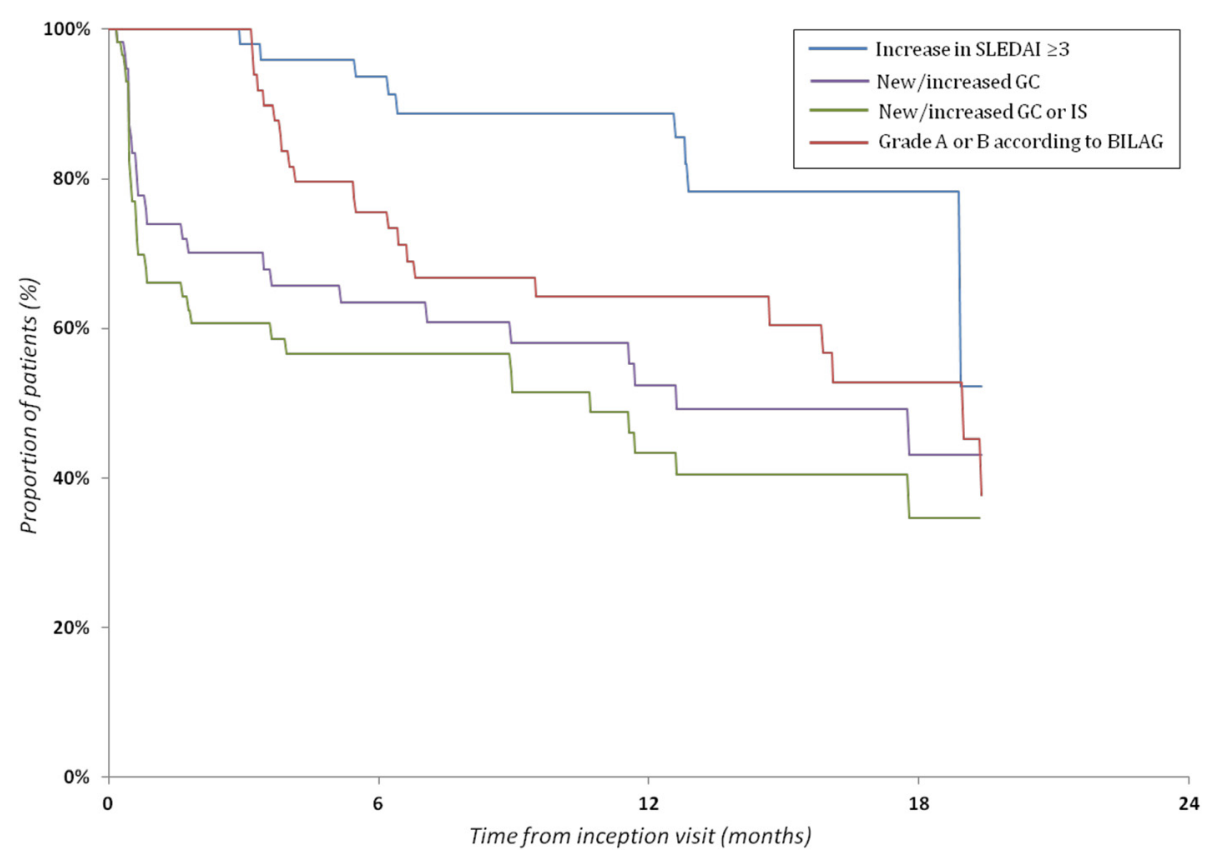

Fig. 3 Differential in time to flare according to the definitions of flare used. Systemic Lupus Erythematosus Disease Activity Index 2000 (SLEDAI) and British Isles Lupus Assessment Group disease activity index (BILAG) scores were collected only at prespecified time points (months 0,3 and 6 and then every 6 months; however, the graphed points are exact dates when the per-protocol visits actually occurred), and reflected only the systemic lupus erythematosus activity within a maximum 30-day time window before each visit. Changes in glucocorticoids and/or immunosuppressive therapy were tracked using the source documentation, and thus these data have finer granularity in time (analysis with the Kaplan-Meier approach for survival estimation). GC glucocorticoids, IS immunosuppressants

Fig. 3 illustrate the dynamics of the sensitivity of different flare definitions in our cohort study. SLEDAI-2 K and BILAG-2004 scores were collected only at prespecified time points (months 0,3 and 6 and then every 6 months) and reflected only the SLE activity within a maximum 30-day time window before each visit. At each per protocol visit, however, the study nurse, by using the source documentation, could retrospectively track any changes in immunosuppressive therapy that had happened since the previous visit, and thus these data have finer granularity in time and reflect more comprehensively the course of the disease. Owing to the size of our

Table 3 Performance of baseline tenascin-C levels to predict disease flares (Cox proportional hazards analysis)

\begin{tabular}{|c|c|c|c|c|}
\hline \multirow[b]{2}{*}{ Flare definition } & \multicolumn{2}{|c|}{ Univariate analyses } & \multicolumn{2}{|c|}{ Age and sex adjusted analyses } \\
\hline & $\mathrm{HR}(95 \% \mathrm{Cl})$ & $p$ Value & $\mathrm{HR}(95 \% \mathrm{Cl})$ & $p$ Value \\
\hline \multicolumn{5}{|l|}{ Tenascin as continuous variable } \\
\hline (i) New/increased GC & $1.39(1.11-1.73)$ & 0.004 & $1.37(1.11-1.70)$ & 0.004 \\
\hline (ii) New/increased GC or IS & $1.25(1.02-1.52)$ & 0.028 & $1.23(1.01-1.49)$ & 0.035 \\
\hline (iii) Increase in SLEDAl-2 $K \geq 3$ & $1.19(0.87-1.63)$ & 0.277 & $1.21(0.86-1.68)$ & 0.270 \\
\hline (iv) BILAG-2004 flare A or B & $1.10(0.91-1.34)$ & 0.323 & $1.09(0.89-1.32)$ & 0.403 \\
\hline \multicolumn{5}{|c|}{ Tenascin as categorical variable $(>659 \mathrm{ng} / \mathrm{ml})^{\mathrm{a}}$} \\
\hline (i) New/increased GC & $3.77(1.60-8.88)$ & 0.002 & $3.57(1.48-8.59)$ & 0.005 \\
\hline (ii) New/increased GC or IS & $2.45(1.10-5.46)$ & 0.028 & $2.23(0.98-5.08)$ & 0.056 \\
\hline (iii) Increase in SLEDAl-2 $K \geq 3$ & $1.42(0.28-7.21)$ & 0.672 & $1.52(0.27-8.64)$ & 0.636 \\
\hline (iv) BILAG-2004 flare A or B & $1.74(0.75-4.04)$ & 0.197 & $1.64(0.70-3.88)$ & 0.257 \\
\hline
\end{tabular}

BILAG-2004 British Isles Lupus Assessment Group disease activity index, Cl confidence interval, HR hazard ratio, IS immunosuppressants, GC glucocorticoids, SLEDAI-2 K Systemic Lupus Erythematosus Disease Activity Index 2000

${ }^{a}$ The threshold value of $659 \mathrm{ng} / \mathrm{ml}$ for tenascin-C (TNC) was generated using receiver operating characteristic curve analysis of the relationship between active systemic lupus erythematosus (SLEDAI- $\mathrm{K} \geq 6$ ) and baseline TNC

Boldface type indicates statistically significant values 


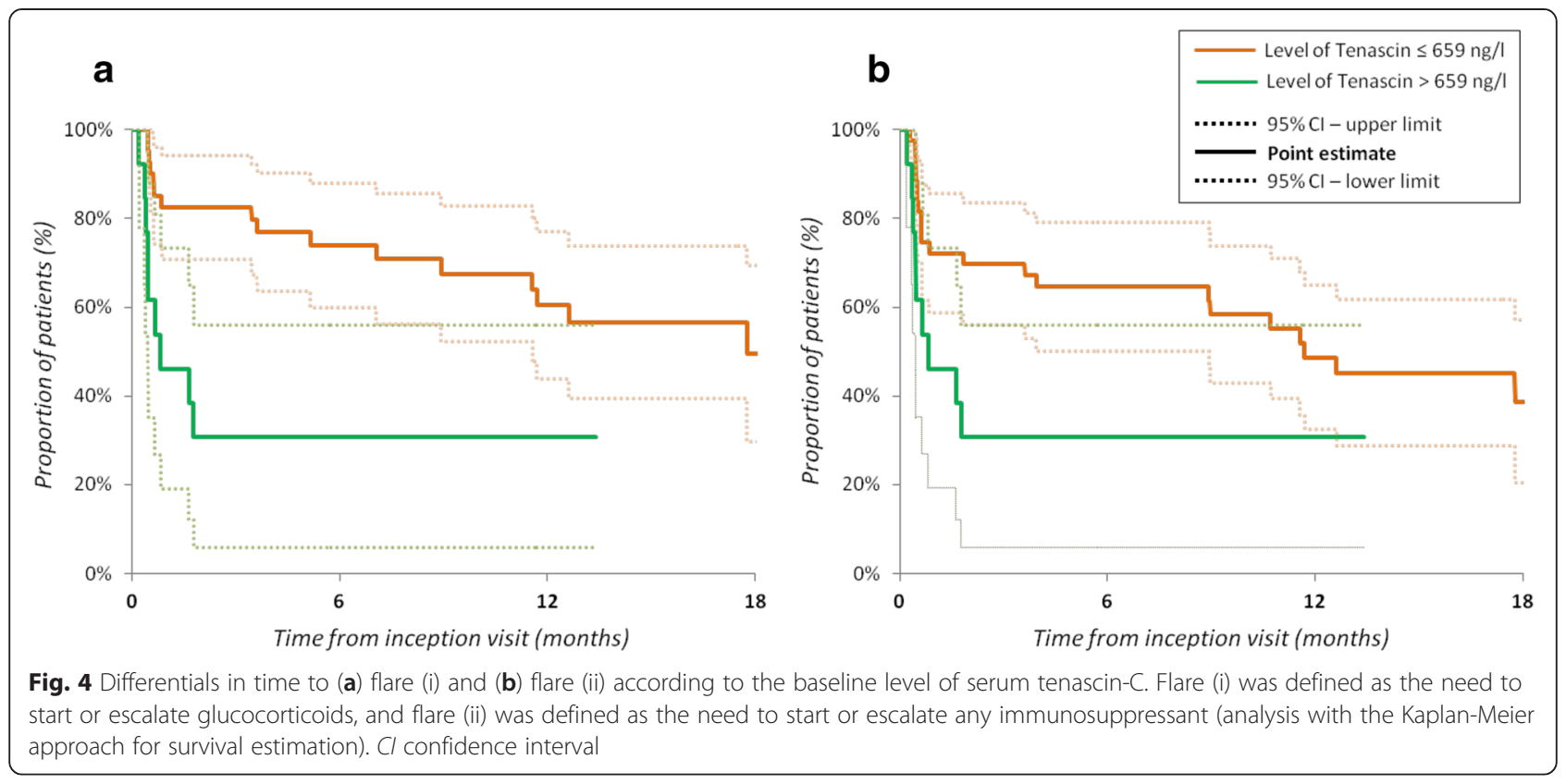

study sample, we preferred to have a more sensitive tool. Hence, our primary definition of the flares was based on the recorded new start or dose escalation of GC. Because GC are still considered to be the most effective treatment for SLE, the need to start or escalate GC seems to be both a sensitive and a pragmatic outcome measurement of a disease flare. However, the addition of an immunosuppressant may not always mirror an increase in SLE activity; it may also represent a steroidsparing strategy. Flare definitions based on change in the SLEDAI-2 K also have several limitations. For example, the SLEDAI-2 K does not capture mild degrees of activity in some organ systems, does not have descriptors for several types of activity (e.g., haemolytic anaemia) and cannot capture worsening of activity in an organ system descriptor [33]. Consequently, even lower SLEDAI-2 K

Table 4 A comparison of the performance of conventional biomarkers vs. tenascin- $C$ to predict the escalation of glucocorticoids in patients with SLE

\begin{tabular}{|c|c|c|c|c|c|c|}
\hline Cox model & Variable & & & & & \\
\hline Continuous & $\mathrm{AIC}$ & C3 & C4 & Anti-dsDNA & Anti-nucleosome & Tenascin \\
\hline $\mathrm{AIC}$ & & 165.02 & 163.52 & 162.67 & 161.87 & 160.40 \\
\hline C3 & 165.02 & 1.000 & 0.220 & 0.125 & 0.076 & 0.032 \\
\hline C4 & 163.52 & 0.220 & 1.000 & 0.357 & 0.199 & 0.078 \\
\hline Anti-dsDNA & 162.67 & 0.125 & 0.357 & 1.000 & 0.370 & 0.132 \\
\hline Anti-nucleosome antibodies & 161.87 & 0.076 & 0.199 & 0.370 & 1.000 & 0.227 \\
\hline Tenascin & 160.40 & 0.032 & 0.078 & 0.132 & 0.227 & 1.000 \\
\hline Categorical & & Low C3 & Low C4 & Anti-dsDNA+ & Anti-nucleosome+ & Tenascin+ \\
\hline $\mathrm{AIC}$ & & 165.45 & 164.44 & 163.21 & 160.25 & 160.06 \\
\hline Low C3 & 165.45 & 1.000 & 0.314 & 0.134 & 0.023 & 0.020 \\
\hline Low C4 & 164.44 & 0.314 & 1.000 & 0.268 & 0.041 & 0.036 \\
\hline Anti-dsDNA antibodies+ & 163.21 & 0.134 & 0.268 & 1.000 & 0.085 & 0.076 \\
\hline Anti-nucleosome antibodies+ & 160.25 & 0.023 & 0.041 & 0.085 & 1.000 & 0.667 \\
\hline Tenascin+ & 160.06 & 0.020 & 0.036 & 0.076 & 0.667 & 1.000 \\
\hline
\end{tabular}

anti-dsDNA anti-double-stranded DNA

This table compares univariate Cox regression models to predict the escalation of therapy by glucocorticoids based on the Akaike information criterion (AIC). The lower the value of the AIC, the better the fit of the model. Predictors are treated in the upper part of the table as continuous variables and in the lower part of the table as categorical variables. Paired comparisons of the quality of each model are illustrated by their respective $p$ values. The thresholds for conventional biomarkers were reference values, and a cutoff value of $654 \mathrm{ng} / \mathrm{ml}$ for tenascin- $C$ was generated using receiver operating characteristic curve analysis to find the optimal discriminatory threshold to identify patients who would require escalation of glucocorticoids. Boldface type indicates statistically significant values 
cutoffs may have suboptimal sensitivity to capture SLE exacerbations [34, 35], while BILAG-2004 flare definitions may (as in our study) perform somewhat better [36]. Importantly, we observed flares defined by changes in immunosuppressive therapy rather early after the inception visit (i.e., close to the instant of blood sampling for TNC measurement), while relapses defined by SLEDAI- $2 \mathrm{~K}$ or BILAG-2004 could occur no earlier than at the time of the next per-protocol visit. Hence, our finding of a possible predictive value of TNC may in fact relate mainly to very early flares and/or reflect worsening of baseline activity.

There is a paucity of validated SLE biomarkers that simultaneously reflect disease activity and, more important, forecast impending flares. Currently, anti-dsDNA antibodies and complement levels are the only serological parameters that are routinely used as activity-specific biomarkers in SLE patient care. However, these traditional biomarkers are not always appropriate for clinical monitoring, because high levels of anti-dsDNA or low levels of $\mathrm{C} 3 / \mathrm{C} 4$ are persistent in some patients with lupus. Recently, anti-nucleosome antibodies were also suggested as a useful biomarker that may have additional value when evaluated with traditional biomarkers [37].

We compared the value of univariate models based on either conventional biomarkers (i.e., anti-dsDNA or antinucleosome antibodies and $\mathrm{C} 3$ or $\mathrm{C} 4$ ) or TNC to predict disease flare (i). In our cohort, serum TNC levels outperformed traditional biomarkers when treated as both a continuous and a categorical variable. It is fair to mention that we used reference threshold values of the conventional biomarkers, while the threshold value for TNC was generated by a ROC curve analysis for this very purpose using our SLE cohort. However, in daily clinical practice, conventional biomarkers are always interpreted in the context of their reference values; hence, such a comparison with a new investigational biomarker is sensible. We did not compare the role of conventional biomarkers with TNC to discriminate between active versus inactive disease, because anti-dsDNA and complement contribute to the SLEDAI-2 K-2 K score on which our definition of active disease was based.

Some questions remain. TNC was shown to correlate with the degree of activity in some diseases and clinical scenarios [13-15], while in others it appeared to reflect subsequent tissue remodelling or irreversible damage $[18,24,26]$. TNC is probably neither disease-specific nor pathology-specific, but rather a more universal and ubiquitous marker of ongoing tissue injury, although its potential for monitoring lupus nephritis may deserve closer evaluation. Further study in patients with SLE is required to elucidate whether fluctuations in TNC levels may be used to herald early and reversible changes (i.e., activity) or to signal ongoing fibrosis and progressive and/or irreversible tissue damage. Interestingly, some data indicate that GC may suppress TNC expression [38]. We did not find any correlation with the current dose of GC (data not shown), and we were unable to measure the therapeutic response to GC in our cohort owing to the limited follow-up duration. A much larger study would be required to evaluate the predictive role of TNC for long-term organ damage accrual in SLE.

Our study has several strengths. To the best of our knowledge, this study is the largest to investigate the role of TNC in SLE to date. Our results seem to be consistent both cross-sectionally (TNC levels discriminated active from inactive disease and were correlated with the positivity of traditional biomarkers) and longitudinally (TNC levels were predictive of future escalations of immunosuppressive therapy).

The study has several limitations. It was performed cross-sectionally in a single SLE cohort with a limited number of patients. We did not have sufficient followup data to assess the informational value of dynamic changes in TNC levels and their consistency in serial samples. We also used an open rather than predefined time window for a flare to occur during follow-up after TNC sampling because we were not aware of the "right" model to choose based on the (unknown) TNC pathophysiology in SLE.

\section{Conclusions}

We found that circulating levels of TNC aided in the discrimination of patients with SLE with active disease from $\mathrm{HC}$ or patients with no or low disease activity. Moreover, high levels of TNC were associated with an increased risk of the need to start or escalate the dose of GC. Further studies with a larger cohort of patients are required to validate the role of TNC as a useful serum biomarker for monitoring disease activity and predicting flares in patients with SLE.

\section{Additional files}

Additional file 1: Comparison of tenascin-C levels between males and
females, and between female patients and female healthy controls.
(PDF $386 \mathrm{~kb}$ )
Additional file 2: a-g Sensitivity analyses showing cross-sectional
associations between tenascin-C levels and SLE activity in various
organ domains according to SLEDAI-2 K and BILAG-2004 indices at
inception visit. (PDF $853 \mathrm{~kb}$ )
Additional file 3: a Cross-sectional associations between serum
tenascin-C levels and positivity of anti-ds-DNA antibodies at the
inception visit (univariate analysis). b Cross-sectional associations
between serum tenascin-C levels and positivity of anti-nucleosome
antibodies at the inception visit (univariate analysis). (ZIP $46 \mathrm{~kb}$ )

Abbreviations

AIC: Akaike information criterion; ANA: anti-nuclear antibodies; anti-dsDNA: antidouble-stranded DNA antibody; BILAG: British Isles Lupus Assessment Group disease activity index; Cl: confidence interval; c-SLEDAI-2 K: Systemic Lupus Erythematosus Disease Activity Index 2000 clinical items; ELISA: enzyme-linked 
immunosorbent assay; GC: glucocorticoids; HC: healthy controls; HR: hazard ratio: IF: immunofluorescence; IS: immunosuppressants; LIA: line immunoassay; ROC: receiver operating characteristic; SD: standard deviation; SLE: systemic lupus erythematosus; SLEDAI-2 K: Systemic Lupus Erythematosus Disease Activity Index 2000; SLICC/ACR: Systemic Lupus International Collaborating Clinics/American College of Rheumatology; TNC: tenascin-C.

\section{Competing interests}

The authors declare that they have no competing interests.

\section{Authors' contributions}

JZ designed the study and wrote the manuscript draft. MU performed the statistical analysis. HH performed the TNC measurements. JZ, RS, MO, MH, HC, MT, LS and JV substantially contributed to the acquisition and/or interpretation of the presented data. All authors revised the manuscript, and all read and approved the final manuscript.

\section{Acknowledgements}

We thank Milada Lösterová for her excellent work as a study nurse in organizing the conduct of the study. This study was supported by a grant from the Czech Ministry of Health (IGA grant NT 13707).

\section{Author details}

'Institute of Rheumatology, Prague, and Department of Rheumatology, First Faculty of Medicine, Charles University in Prague, Na Slupi 4, Praha 2, 12850 Prague, Czech Republic. ${ }^{2}$ Institute of Biostatistics and Analyses, Masaryk University, Brno, Czech Republic.

Received: 26 August 2015 Accepted: 13 November 2015 Published online: 25 November 2015

\section{References}

1. Illei GG, Tackey E, Lapteva L, Lipsky PE. Biomarkers in systemic lupus erythematosus: II. Markers of disease activity. Arthritis Rheum. 2004;50:2048-65.

2. Liu CC, Manzi S, Ahearn JM. Biomarkers for systemic lupus erythematosus: a review and perspective. Curr Opin Rheumatol. 2005;17:543-9.

3. Esdaile JM, Abrahamowicz M, Joseph L, MacKenzie T, Li Y, Danoff D. Laboratory tests as predictors of disease exacerbations in systemic lupus erythematosus: why some tests fail. Arthritis Rheum. 1996;39:370-8.

4. Midwood KS, Hussenet T, Langlois B, Orend G. Advances in tenascin-C biology. Cell Mol Life Sci. 2011;68:3175-99.

5. Midwood KS, Orend G. The role of tenascin-C in tissue injury and tumorigenesis. J Cell Commun Signal. 2009;3:287-310.

6. Chiquet-Ehrismann $\mathrm{R}$, Chiquet $\mathrm{M}$. Tenascins: regulation and putative functions during pathological stress. J Pathol. 2003;200:488-99.

7. Udalova IA, Ruhmann M, Thomson SJ, Midwood KS. Expression and immune function of tenascin-C. Crit Rev Immunol. 2011;31:115-45.

8. Midwood K, Sacre S, Piccinini AM, Inglis J, Trebaul A, Chan E, et al. Tenascin-C is an endogenous activator of Toll-like receptor 4 that is essential for maintaining inflammation in arthritic joint disease. Nat Med. 2009;15:774-80.

9. Goh FG, Piccinini AM, Krausgruber T, Udalova IA, Midwood KS Transcriptional regulation of the endogenous danger signal tenascin-C: a novel autocrine loop in inflammation. J Immunol. 2010;184:2655-62.

10. Ruhmann M, Piccinini AM, Kong PL, Midwood KS. Endogenous activation of adaptive immunity: tenascin- $C$ drives interleukin-17 synthesis in murine arthritic joint disease. Arthritis Rheum. 2012;64:2179-90.

11. Sumioka T, Fujita N, Kitano A, Okada Y, Saika S. Impaired angiogenic response in the cornea of mice lacking tenascin C. Invest Ophthalmol Vis Sci. 2011;52:2462-7.

12. Hisatomi K, Sakamoto N, Mukae H, Hayashi T, Amenomori M, Ishimoto H, et al. Elevated levels of tenascin-C in patients with cryptogenic organizing pneumonia. Intern Med. 2009:48:1501-7.

13. Tanaka H, El-Karef A, Kaito M, Kinoshita N, Fujita N, Horiike S, et al. Circulating level of large splice variants of tenascin-C is a marker of piecemeal necrosis activity in patients with chronic hepatitis C. Liver Int. 2006:26:311-8

14. Riedl S, Tandara A, Reinshagen M, Hinz U, Faissner A, Bodenmüller $H$, et al. Serum tenascin- $C$ is an indicator of inflammatory bowel disease activity. Int J Colorectal Dis. 2001;16:285-91.
15. Imanaka-Yoshida K, Hiroe M, Yasutomi Y, Toyozaki T, Tsuchiya T, Noda N, et al. Tenascin-C is a useful marker for disease activity in myocarditis. J Pathol. 2002;197:388-94.

16. Wallner K, Li C, Shah PK, Fishbein MC, Forrester JS, Kaul S, et al. Tenascin-C is expressed in macrophage-rich human coronary atherosclerotic plaque. Circulation. 1999;99:1284-9.

17. Catalán V, Gómez-Ambrosi J, Rodríguez A, Ramírez B, Rotellar F, Valentí V, et al. Increased tenascin C and Toll-like receptor 4 levels in visceral adipose tissue as a link between inflammation and extracellular matrix remodeling in obesity. J Clin Endocrinol Metab. 2012;97:E1880-9.

18. Page TH, Charles PJ, Piccinini AM, Nicolaidou V, Taylor PC, Midwood KS. Raised circulating tenascin-C in rheumatoid arthritis. Arthritis Res Ther. 2012;14:R260

19. Shukla A, Gaur P, Aggarwal A. Tenascin-C levels, a Toll-like receptor 4 ligand, in enthesitis-related arthritis category of juvenile idiopathic arthritis: a crosssectional and longitudinal study. J Rheumatol. 2015;42:891-6.

20. Hochberg MC. Updating the American College of Rheumatology revised criteria for the classification of systemic lupus erythematosus. Arthritis Rheum. 1997:40:1725.

21. Gladman DD, Ibanez D, Urowitz MB. Systemic Lupus Erythematosus Disease Activity Index 2000. J Rheumatol. 2002;29:288-91.

22. Isenberg DA, Rahman A, Allen E, Farewell V, Akil M, Bruce IN, et al. BILAG 2004: development and initial validation of an updated version of the British Isles Lupus Assessment Group's disease activity index for patients with systemic lupus erythematosus. Rheumatology (Oxford). 2005;44:902-6.

23. Abrahamowicz M, Fortin PR, du Berger R, Nayak V, Neville C, Liang MH. The relationship between disease activity and expert physician's decision to start major treatment in active systemic lupus erythematosus: a decision aid for development of entry criteria for clinical trials. J Rheumatol. 1998:25:277-84.

24. Inoue K, Jinnin M, Hara Y, Makino K, Kajihara I, Makino T, et al. Serum levels of tenascin-C in collagen diseases. J Dermatol. 2013;40:715-9.

25. Truong LD, Pindur J, Barrios R, D'Agati V, Lechago J, Suki W, et al. Tenascin is an important component of the glomerular extracellular matrix in normal and pathologic conditions. Kidney Int. 1994;45:201-10.

26. Brissett M, Veraldi KL, Pilewski JM, Medsger Jr TA, Feghali-Bostwick CA. Localized expression of tenascin in systemic sclerosis-associated pulmonary fibrosis and its regulation by insulin-like growth factor binding protein 3 . Arthritis Rheum. 2012;64:272-80.

27. Imanaka-Yoshida K. Tenascin-C in cardiovascular tissue remodeling: from development to inflammation and repair. Circ J. 2012;76:2513-20.

28. Jakovcevski I, Miljkovic D, Schachner M, Andjus PR. Tenascins and inflammation in disorders of the nervous system. Amino Acids. 2013;44:1115-27.

29. Jyo Y, Sasaki T, Nomura S, Tamai H, Kawai S, Osawa G, et al. Expression of tenascin in mesangial injury in experimental glomerulonephritis. Exp Nephrol. 1997:5:423-8.

30. Masaki T, Yorioka N, Taniguchi Y, Oda H, Yamakido M. Tenascin expression may reflect the activity and chronicity of human IgA nephropathy. Clin Nephrol. 1998;50:205-13.

31. Hörstrup JH, Gehrmann M, Schneider B, Plöger A, Froese P, Schirop T, et al Elevation of serum and urine levels of TIMP-1 and tenascin in patients with renal disease. Nephrol Dial Transplant. 2002;17:1005-13.

32. Liang MH, Simard JF, Costenbader K, Dore BT, Ward M, Fortin PR, et al. Methodologic issues in the validation of putative biomarkers and surrogate endpoints in treatment evaluation for systemic lupus erythematosus. Endocr Metab Immune Disord Drug Targets. 2009;9:108-12.

33. Petri M, Buyon J, Kim M. Classification and definition of major flares in SLE clinical trials. Lupus. 1999;8:685-91.

34. Yee CS, Farewell VT, Isenberg DA, Griffiths B, Teh LS, Bruce IN, et al. The use of Systemic Lupus Erythematosus Disease Activity Index-2000 to define active disease and minimal clinically meaningful change based on data from a large cohort of systemic lupus erythematosus patients. Rheumatology (Oxford). 2011;50:982-8.

35. American College of Rheumatology Ad Hoc Committee on Systemic Lupus Erythematosus Response Criteria. The American College of Rheumatology response criteria for systemic lupus erythematosus clinical trials: measures of overall disease activity. Arthritis Rheum. 2004;50:3418-26.

36. Yee CS, Isenberg DA, Prabu A, Sokoll K, Teh LS, Rahman A, et al. BILAG-2004 index captures systemic lupus erythematosus disease activity better than SLEDAI-2000. Ann Rheum Dis. 2008;67:873-6. 
37. Li T, Prokopec SD, Morrison S, Lou W, Reich H, Gladman D, et al. Antinucleosome antibodies outperform traditional biomarkers as longitudinal indicators of disease activity in systemic lupus erythematosus. Rheumatology (Oxford). 2015;54:449-57.

38. Cella N, Chiquet-Ehrismann R, Hynes NE. Lactogenic hormones and tenascin-C regulate C/EBPa and $\beta$ in mammary epithelial cells. J Cell Biochem. 2000;76:394-403.

Submit your next manuscript to BioMed Central and we will help you at every step:

- We accept pre-submission inquiries

- Our selector tool helps you to find the most relevant journal

- We provide round the clock customer support

- Convenient online submission

- Thorough peer review

- Inclusion in PubMed and all major indexing services

- Maximum visibility for your research 